\title{
Muss (auch) die Philosophie populär werden?
}

\author{
Hâtons-nous de rendre la philosophie populaire! \\ Denis Diderot \\ Festina lente \\ Aldo Manuzio
}

\section{Die Herausforderung der Philosophie}

Der große Wunsch der Aufklärung war die Verbreitung des Wissens und damit einhergehend die Errichtung einer gerechteren Gesellschaft. Das war die Zeit der philosophes. Wir bleiben bis heute vielfach auf die Idee der Gerechtigkeit fixiert, wissen aber nicht unbedingt, was sie für das Wissen und die Philosophie noch bedeuten kann. Und wenn wir uns trotz vieler Schwierigkeiten nach wie vor für ein derartiges Projekt erwärmen: Sollen wir uns wirklich beeilen, die Philosophie populär zu machen?

Die Welt wird immer komplexer, differenzierter, bunter - wir sehen immer mehr Bezüge, Vernetzungen, Horizonte (die sogenannte Globalisierung) und drohen dabei leicht die Übersicht zu verlieren. Auch was wir tagtäglich an technischem Gerät benutzen, verstehen wir kaum. Wir erwarten von solchen Gegenständen, dass sie einfach zu gebrauchen sind. Wir wollen nicht wissen, wie sie funktionieren, sondern mit ihnen unmittelbar bestimmte Wirkungen erzielen, meist indem wir auf einen Knopf drücken oder auf etwas klicken.

Die Welt wird komplexer zwischen dem Globalen und dem Lokalen, dem Politischen und dem Wirtschaftlichen, dem Virtuellen und dem Handhabbaren. Das Bedürfnis nach einem einfachen Bild und nach einfachen Lösungen wächst. Wenn schon die Politik die Komplexität der gesellschaftlichen Situation eines Landes ihren Wählern nicht mehr erklären kann, darf man dann eigentlich

\footnotetext{
Anmerkung: Diese Ausführungen sind ein Versuch, die Gedanken und Bedenken über das Verhältnis der Philosophie zu ihrer Sprache, die in dem Buch Geteilte Ideen. Philosophische Versuche, den Leser zum Verstehen zu bringen vorgelegt worden sind (Thouard 2016), darzustellen und weiterzuführen. Das Problem beschäftigt mich seit dem Band Popularité de la philosophie, den ich 1995 zusammen mit Philippe Beck herausgegeben habe (Beck \& Thouard 1995). Auf Gelehrsamkeit wurde weitgehend verzichtet, der Popularität hingegen Tribut gezollt.
}

Ә Open Access. (C) 2019 Denis Thouard, publiziert von De Gruyter. (cc)BY-NC-ND Dieses Werk ist lizenziert unter der Creative Commons Attribution-NonCommercial-NoDerivatives 4.0 Lizenz.

https://doi.org/10.1515/9783110676631-003 
fordern, dass die Wissenschaft, die weit weniger übersichtlich scheint, so anschaulich oder vereinfachend erklärt wird, dass sie allen zugänglich ist? Lässt sich die Wissenschaft überhaupt popularisieren?

Das Problem stellt sich für die Philosophie in besonderer und verschärfter Form, weil sie mit der Religion den Anspruch teilt, eine Rede über das Ganze zu liefern. Sie hat aber im Vergleich zu dieser den großen Nachteil, dass sie traditionell nicht an den Glauben appelliert, sondern an den Verstand, sie will verstanden werden. Nur ,geglaubte‘ Philosophie ist keine Philosophie. Aber indem sie sich diesem Anspruch mit mehr oder weniger Erfolg verschreibt, ist die Philosophie eine besondere Disziplin geworden. Sie hat eine bestimmte Sprache, bestimmte Themen und Gegenstände, eine eigene institutionelle Organisation, eine Geschichte.

Die fortschreitende Differenzierung der Gesellschaften verschärft zwangsläufig die Spannung zwischen dem Ganzheitsanspruch und der tatsächlichen Spezialisierung einer akademischen Disziplin. Wie kann der Wunsch nach Orientierung, nach Lebensführung, nach Sinngebung oder nach Hilfe von der Philosophie als einer akademischen Disziplin erfüllt werden? Und wenn sie es versuchen würden, wie könnten Philosophen jemals sicher sein, dass sie auch verstanden werden?

Trotz ihrer disziplinären Zurückgezogenheit sind die Philosophen in der Öffentlichkeit nicht völlig abwesend. Einige schaffen es sehr wohl, sich einem breiteren Publikum zu empfehlen. Die Frage ist aber, ob sie dies noch als Philosophen tun oder bereits als Intellektuelle, als Stars, als moralische Autoritäten, als engagierte Aktivisten. Die Gestaltung möglicher Öffentlichkeiten oder Kreise ist freilich je nach Gesellschaft eine andere.

Geradezu topisch ist die Ferne des Philosophen vom alltäglichen Leben, von der Gesellschaft. Die von Platon nacherzählte Geschichte von Thales, der in den Brunnen fällt, als er den Himmel beobachtete, ist bis heute paradigmatisch.

Wie auch den Thales, o Theodoros, als er, um die Sterne zu beschauen, den Blick nach oben gerichtet in den Brunnen fiel, eine artige und witzige thrakische Magd soll verspottet haben, daß er, was am Himmel wäre, wohl strebte zu erfahren, was aber vor ihm läge und zu seinen Füßen, ihm unbekannt bliebe, - mit diesem nämlichen Spotte nun reicht man noch immer aus gegen alle, welche in der Philosophie leben. Denn in der Tat, ein solcher weiß nichts von seinem Nächsten und Nachbar, nicht nur nicht, was er betreibt, sondern kaum, ob er ein Mensch ist oder etwa irgend ein anderes Geschöpf. ${ }^{1}$

Von der Magd wird er zu Recht ausgelacht. An diesem Lachen kann man die ganze Distanz und das ganze Missverständnis zwischen den Philosophen und

1 Platon 1958. 
dem ,Volk“ ermessen. Dem „Lachen der Thrakerin“ hat Hans Blumenberg ein feinsinniges Buch gewidmet. Der theoretische Blick droht immer, das Naheliegende zu vernachlässigen, was sich allerdings unmittelbar rächt. ${ }^{2}$

Die kleine Komödie, die der Theaitetos uns erzählt, liefert aber mitnichten die letzte Weisheit über die Unverständlichkeit der Philosophie.

Von Anfang an war das Praktizieren der Philosophie eine Tätigkeit, die nur einem bestimmten kleinen Kreis zukam. Wenigstens Geometer musste man sein, um aufgenommen zu werden. In dieser Spezialisierung musste man gleichwohl in der Lage sein, sich an andere zu richten, zuerst an die Beginnenden, an die Anfänger. Man musste kommunizieren, aber nicht die Wahrheiten, die man suchte, sondern die Art und Weise, wie man zu solchen gelangen konnte. Es wurde eine Form der Verständigung angestrebt, die gewissermaßen als Filter wirkte zwischen denen, die sich Eingang verschaffen konnten, und denen, die sich damit schwer taten.

Man hat dieses Problem unter unterschiedlichen Begriffsbestimmungen $\mathrm{zu}$ behandeln versucht: In der Antike unter dem Gegensatz zwischen dem Esoterischen, das für eine kleine Gruppe bestimmt war, und dem Exoterischen, das sich einem zwar breiteren, aber doch noch begrenzten Publikum verschreiben sollte. Diese Unterscheidung blieb allerdings von untergeordneter Bedeutung für unser Verständnis der antiken Philosophie: Man kennt Platon aus seinen exoterischen, Aristoteles aus seinen esoterischen Schriften, aber man meint den einen ebenso gut (oder ebenso schlecht) zu kennen wie den anderen.

In der Neuzeit und bis heute wurde die Frage nach der Vermittlung der Philosophie wieder besonders akut, nicht zuletzt, weil sich demokratische Prinzipien zusehends zu behaupten vermochten. Man fragte nicht mehr, ob es möglich sei, die Philosophie allgemein zu verbreiten, sondern forderte es förmlich - man denke an die eingangs zitierten Worte von Diderot. Die Philosophie sollte sich an alle richten und träumte sogar davon, von allen verstanden zu werden. Das ist das Programm der Aufklärung, das bei Fichte in dem Anspruch kulminierte, „den Leser zum Verstehen zu zwingen“. 3

Beide Perspektiven unterscheiden sich grundlegend. Wenn das Publikum im demokratischen Zeitalter für sein Recht auf Zugang zu den Wissenschaften und den Ideen kämpft, kann ein Philosoph sich wünschen, die Richtigkeit seines Systems allen aufzuzwingen. Wenn er sich im Besitz der Wahrheit wähnt warum sollte er mit dieser geizen? Aber wie kann man überhaupt sicher sein,

2 Blumenberg 1987: 14: „Von Plato her gesehen, ist in jener Komödie am Brunnenrand [...] der Zusammenstoß von Welten, von Wirklichkeitsbegriffen thematisch, deren Unverständigkeit gegeneinander die Erscheinung der Lächerlichkeit [. . .] annehmen kann.“

3 Fichte 1801. 
dass man die, oder auch nur eine, Wahrheit jemals erreicht hat? Und ist diese Vorstellung für die Philosophie konstitutiv? Es ist gerade die Geschichte bestimmter Philosophen zu ihrem (oft imaginierten) Publikum oder Adressatenkreis, die zeigt, dass diese Frage alles andere als leicht $\mathrm{zu}$ beantworten ist.

Dafür gilt es, zunächst einen Blick auf die Vorgeschichte der Popularphilosophie in der Frühen Neuzeit zu werfen, zumal dort, wo sich diese mit Problemen der Zweisprachigkeit oder Diglossie konfrontiert sieht. Das Programm der Popularphilosophie erweist sich dann in einem zweiten Schritt als ein genuin aufklärerisches, was man nicht zuletzt an der Schlüsselstellung von Kant ablesen kann. Kant hat maßgeblich die praktische Philosophie ins Zentrum gestellt und dabei die ganze Architektonik der Philosophie als System neu gedacht. Dass er zentrale Spannungen innerhalb der vorherigen Philosophiegeschichte zu verabschieden suchte, bewirkte in seinem Fall eine völlig neue Sicht auf die Darstellung der Philosophie, die allerdings weniger Kant selbst als vielmehr seine Nachfolger wie Schelling, Hegel, Schleiermacher oder Schlegel beschäftigen sollte. Hegel wird dieses Problem vielfach auf die Spitze treiben.

\section{Von der Vulgarisierung zur Popularisierung}

Wenn wir uns zunächst einer anderen historischen Periode widmen, nämlich der Frühen Neuzeit und der viel gepriesenen Res publica litteraria, erblicken wir zwar einen internationalen Kreis, doch bleibt dieser ausgesprochen elitär. Man schrieb, von Erasmus bis zu Clericus oder Muratori, natürlich meist auf Latein. Aber die allmähliche Behauptung einer Philosophie in volgare, in den jeweiligen Landessprachen Europas, hat dem Lateinischen zusehends zugesetzt, so dass es im Laufe des 19. Jahrhunderts zumindest in philosophischen Texten kaum mehr verwendet wurde.

Die Situation der Zweisprachigkeit hat unsere wissenschaftliche Vorgeschichte geprägt. Sie war in Griechenland unbekannt, was vielleicht die immer wiederkehrende Sehnsucht nach der griechischen Antike erklärt. Allerdings lassen sich in dieser sehr wohl bereits unterschiedliche Dialekte und stilistische Formate beobachten. In Rom begann dann aber etwas völlig anderes: Die gelehrte Welt sprach und schrieb dort auf Griechisch, die politische Welt hingegen auf Latein. Der Kampf Ciceros und anderer um die Bildung einer Wissenschaftssprache oder einer philosophischen Sprache ist bekannt. Er endete so erfolgreich, dass sich in der Wissenschaft mehr noch als in der Dichtung seit der Etablierung der Nationalsprachen eine Art Diglossie jahrhundertelang $\mathrm{zu}$ behaupten vermochte: 
Im 14. Jahrhundert schrieb Boccaccio seine philosophischen Werke auf Latein, die anderen auf Italienisch.

Diese Diglossie blieb nicht auf die Renaissance beschränkt. Sie lässt sich bis weit in die Aufklärung hinein verfolgen, in der sich neben dem Lateinischen differenzierte philosophische Nationalsprachen herausbildeten. Trotzdem bleibt der Unterschied der Register offenkundig, sei es bei Bacon oder Hobbes (De Cive versus Leviathan) oder bei Descartes, der den Discours de la méthode auf Französisch schreibt, damit ,alle ' ihn lesen können, für die Meditationes aber am Lateinischen festhält und diese nicht einmal selbst übersetzt. Die erwähnten Schriften sind an jeweils unterschiedliche Adressaten gerichtet. Die Notwendigkeit einer ,leichten' Fassung in der Nationalsprache wird in der Regel mit der Allgemeinheit der Vernunft in Beziehung gesetzt, was sich bereits am Incipit des Discours zeigt: „Le bon sens est la chose du monde la mieux partagée“.

Nun scheint die Popularisierung der Lehre die Produktion der Ideen aber zusehends und nachhaltig unter Druck zu setzen. Die verständlichste Formulierung einer Theorie wird allmählich als die wichtigste betrachtet. Das Lateinische hingegen bleibt akademischen Konventionen und Konzessionen vorbehalten. Die dissertatio muss man gemacht haben, um der akademischen Welt zugehörig zu sein, sie verliert aber inhaltlich an Gewicht. Bis ins 20. Jahrhundert hinein wurden noch Dissertationen auf Latein geschrieben, sie haben dabei aber bezeichnenderweise an Umfang wie an Relevanz verloren.

Die Struktur der Netzwerke der République des lettres sowie die Beständigkeit akademischer Usancen an der Universität lässt beide Sprachen und beide Darstellungsformen gelten: die Philosophie richtet sich einerseits an die Kollegen, andererseits wird aber mitunter zusätzlich eine zugänglichere Fassung aufgeboten, die der angestrebten Allgemeingültigkeit der Philosophie Rechnung trägt.

Die Entwicklung, die sich hier beobachten lässt, ist eine langsame Verschiebung des philosophischen Gewichts von der gelehrten Sprache zur jeweiligen Nationalsprache. Diese Verschiebung ist keine rein technische Angelegenheit, sondern zeugt darüber hinaus von einem neuen Anspruch. Die Form, die für ein breites Publikum gewählt wurde, also die populäre Darstellung, die sich an ein Volk richtete, übt jetzt auf den Inhalt der Philosophie selbst einen erheblichen Einfluss, wo nicht sogar Zwang aus. Das Volk erscheint nun mehr und mehr als eine konkrete Form von ,Allgemeinheit‘. Populariter bedeutet nicht mehr nur die Anpassung an ein bestimmtes Publikum, ad populum, sondern enthält nach und nach den Anspruch, für alle möglichen Adressaten gültig und zugänglich zu sein. Es ist auch die Botschaft der Aufklärung, daran zu erinnern, dass die Philosophie alle Welt betrifft (oder, nach Kants Bestimmung des Weltbegriffs der 
Philosophie, das, „was jedermann notwendig interessiert“). ${ }^{4}$ Auch in Deutschland, wo der Gegensatz von ,Schul-“ und ,Weltphilosophie‘ meist ausgeprägt war, zeichnet sich diese Tendenz ab. Sie wird schließlich die Ablösung der einen durch die andere bewirken.

Die allgemeine Mitteilbarkeit wirkt also auf die Ansprüche der Philosophie zurück, indem sie sich an diesem Prüfstein nunmehr messen (lassen) muss. Mit Kant:

Wenn aber, nach Shaftesbury's Behauptung, es ein nicht zu verachtender Probierstein für die Wahrheit einer (vornehmlich praktischen) Lehre ist, wenn sie das Belachen aushält, so müßte wohl an den kritischen Philosophen mit der Zeit die Reihe kommen, zuletzt und auch am besten zu lachen. ${ }^{5}$

So hätte am Ende doch die thrakische Magd recht gehabt. Das Lachen hat den Vorrang der Theorie durch den der Praxis ersetzt.

Mehr als eine rein innere Dialektik im jeweiligen Sprachgebrauch ist die Durchsetzung des Praktischen vor dem Theoretischen im Laufe der Aufklärung das, was die Frage nach dem Adressaten der Philosophie grundsätzlich anders stellt. Eine gewisse Rhetorisierung der Philosophie lässt sich bereits in der Renaissance beobachten, als eine Vielzahl von literarischen Gattungen wie Essays und Dialoge philosophisches Allgemeingut wurde. Aber mit der Aufklärung erst wird die Überzeugung topisch, dass die Vernunft, als praktische Vernunft, alle betrifft, und dass folglich alle Zugang zu ihren Produkten haben dürfen, so sehr die politische Zensur oder auch die Hochnäsigkeit der Gelehrten sich dagegen auch stemmen mögen.

Das Verständnis der Philosophie hat sich seit der Aufklärung dahingehend geändert, dass ihre allgemeine Mitteilung und Verbreitung, letztlich ihre Verwirklichung, zu einem unabdingbaren Teil ihrer Aufgabe wurde.

\section{Popularisierung als Imperativ}

Die zu Beginn zitierten Worte Diderots über eine nunmehr unverzichtbare Popularisierung der Philosophie stammen aber aus einem anderen Kontext. Die Schrift De l'interprétation de la nature, die 1754 erschien, ist eine Abhandlung über die Methode der experimentellen Wissenschaften und bewegt sich im

4 Kant 1998: A 840/B 868: Weltbegriff heißt hier derjenige, der das betrifft, was jedermann notwendig interessiert.

5 Kant 2009: VI, 209. 
Fahrwasser von Bacon. Philosophie hieß Naturphilosophie, ihre Verbreitung war ganz im Sinn des Projekts der Enzyklopädie. Trotzdem setzte Diderot damit einen rhetorischen Maßstab für die gesamte Philosophie seiner Zeit. Er hatte das Motto der Aufklärung, wörtlich genommen, so formuliert: „Hâtons-nous de rendre la philosophie populaire!“ Bemühen wir uns, oder genauer, beeilen wir uns, die Philosophie populär zu machen! ${ }^{6}$

Diderot erklärt in diesem Kontext auch, was er mit der Enthüllung der Wahrheit genau meint:

Das Enthüllen an sich reicht noch nicht aus; die Enthüllung muß auch vollständig und klar sein. Es gibt eine Art von Dunkelheit, welche man die Affectation der großen Meister nennen könnte. Man liebt, zwischen dem Volk und der Natur einen Schleier zu ziehen. ${ }^{7}$

Nicht nur dieses Vorhaben Diderots scheint bemerkenswert, neu sind auch die Energie und das Engagement, mit denen er es ausruft. Vielleicht sollte man sich dennoch nicht allzu sehr beeilen, zumal man nicht unbedingt weiß, was Philosophie überhaupt ist. Immerhin wurde der kommunikative Imperativ Diderots bald in Deutschland rezipiert, wo er erheblich zur Erscheinung der sogenannten Popularphilosophie beitrug.

Johann August Ernesti (1707-1781), Sohn eines Pastors, selbst Theologe und Philologe, war seit 1742 Professor für Alte Literatur an der Universität Leipzig. Ernesti hat zahlreiche antike Texte ediert, eine große Vorliebe hatte er für Cicero. In einer 1745 an der Thomasschule gehaltenen Rede mit dem Titel De philosophia populari nimmt Ernesti nicht nur Diderots Parole auf, er integriert sie auch in das Programm einer anderen Art der Philosophie, die sich an Sokrates anlehnt, „qui de coelo deductam philosophiam in urbibus collocavit, et in curias, in forum, in domos etiam privatas introduxit“, und dabei auch auf Cicero rekurriert, der die griechischen Philosopheme in Rom eingebürgert hatte. ${ }^{8}$ Ernesti folgt Cicero, indem er Diderot in die damalige Wissenschaftssprache übersetzt: studeamus philosophiam popularem efficere!

Christian Garve (1742-1798) hat wie kein anderer das Programm einer Popularphilosophie durchgeführt und verkörpert. Er schrieb konsequent auf Deutsch. Der Essay wurde dabei zur philosophischen Leitgattung und die Klarheit des Ausdrucks das geeignete Mittel, Philosophie zu betreiben. Von der Popularität des Vortrags kann als Beschreibung von Garves eigener Darstellung und Methode

6 Diderot 1961: 216, Abschnitt XL.

7 „Ce n'est pas assez de révéler; il faut encore que la révélation soit entière et claire. Il est une sorte d'obscurité que l'on pourrait définir l'affection des grands maîtres. C'est un voile qu'ils se plaisent à tirer entre le peuple et la nature.“ (Ebd.: 215; Übers. U. Kunzmann)

8 Ernesti 1767: 190. 
gelten. ${ }^{9}$ Diese zielen sehr bewusst auf die Bildung einer öffentlichen Meinung, die es damals im strengen Sinn noch gar nicht gab. Sein Ziel ist wohl eine aufgeklärte Republik, die einer bürgerlichen Öffentlichkeit entspricht.

Es wird der jetzt herrschenden Partey der Philosophen [. . . ] nicht entgangen seyn, daß ich ein populärer Philosoph, im schlimmsten Sinne des Wortes, oder vielmehr, daß ich ein Prediger des allgemeinen Menschensinnes - des Feindes aller ächten Philosophie, sey. ${ }^{10}$

Das Plädoyer für Klarheit und Verständlichkeit nimmt bei Garve einen besonders pathetischen Ton an, denn es kollidiert ständig mit der Unmöglichkeit der eigenen Realisierung. Obwohl er zweifellos einen scharfen analytischen Verstand besitzt, der bestimmte moralische oder soziale Erscheinungen feinsinnig zu beschreiben versteht, scheint Garve oft der Sinn für Zusammenhänge und Beziehungen zu fehlen. Bei umfassenden Konstruktionen hört sein Verständnis auf, und er durchschaut offenbar nicht immer, was er tut. Er leidet bereits unter den Änderungen einer fortschreitenden philosophischen Arbeitsteilung, die die neue Welt, an der er mitwirkt, überhaupt erst ermöglicht und sein eigenes Philosophieren sicher auch fördert. Garve konzentriert sich deshalb auf die Form der Allgemeinheit.

Die Rhetorik der Klarheit zeigt dennoch gemeinsame Züge bei allen Philosophen, die sich auf eine Evidenz der Vernunft berufen. Wenn das, was klar ist, zugleich unhintergehbar auch wahr ist, muss der Hörer oder Leser notwendig von der Argumentation der Vernunft überzeugt werden. Die Rhetorik der Philosophen orientiert sich an der Forderung Quintilians, dass die Rede nicht nur verständlich sei, sondern auch so gestaltet, dass keiner sie nicht verstehen könne, sed ne omnino possit non intelligere curandum. ${ }^{11}$ Diese ,Rhetorik der Vernunft ${ }^{*}$ wird typisch für den Anspruch mancher Philosophen, die meinen, sie müssten immer und geradezu zwangsläufig verstanden werden: von Descartes bis Fichte.

\section{Zwischenbemerkung}

Die Popularphilosophie sagt nicht zuletzt etwas über die Erwartungen aus, die wir in sie setzen können. Natürlich kann ,populär hier verschiedene Dinge meinen. Es geht aber im Grunde um die Inkarnation der Philosophie durch das Volk in einem denkbar weiten Sinn. Die Philosophie, die Anspruch auf Allgemeinheit

9 Garve 1796.

10 Garve 1798: 1.

11 Quintilian 1997: 1286. 
erhebt, muss sich auch als allgemeingültig bewähren. Das Mittel dieser Bewährung ist aber ihre Mitteilbarkeit, die zugleich den ,Probierstein' der Freiheit zu denken bildet. $^{12}$ Dieser wechselseitige Zwang des Denkens muss natürlich die Fähigkeit besitzen, das Lachen der ,anderen' auszuhalten.

Inwieweit es möglich ist, einem solchen Anspruch auf Mittelbarkeit konsequent $\mathrm{zu}$ folgen, wird sich noch herausstellen. Ob es überhaupt sinnvoll ist, wurde früh bezweifelt. Man berief sich dabei gerne auf die tiefen Geheimnisse der Philosophie, zitierte Heraklit, wetterte gegen die Verstandesphilosophie. Herder, Hamann, Jacobi bedauern die durch eine Rhetorik der Klarheit verursachte Verarmung. Die Ausdruckspotentialitäten der Sprache würden auf diese Art und Weise regelrecht verkümmern. Die Individualität verblasse, wenn alle sich auf dieselbe Weise verständigen müssen. Einige Philosophen gehen in ihrem Protest gegen die Tyrannei der Klarheit so weit, dass sie sagen können: „Das Mißverstandendste wäre der Versuch des Denkens, sich verständlich zu machen [...]. Die hartnäckigste Gefahr für das Denken ist die Bemühung, verständlich zu sein." ${ }^{13}$

Es geht hier um mehr als einen gleichsam fachinternen Streit, der nur prüfen soll, ob die Allgemeinheit sich mittels einer klaren Rede als Allgemeingültigkeit konstituiert. Es handelt sich um einen zutiefst politischen Streit, der sich um die Frage dreht, ob und inwiefern die Philosophie, die nicht nur einen theoretischen allgemeinen Anspruch hat, sondern seit der Aufklärung eben auch einen praktischen, auf die konkrete Verwirklichung der Gleichheit und Gerechtigkeit unter den Menschen hinwirken kann.

Beide Dimensionen, die theoretische bzw. epistemische und die praktische, haben jeweils eigene Bedingungen und Ziele, die nicht unbedingt zusammenpassen, sondern möglicherweise kollidieren. Vielleicht ist es eine Illusion der Aufklärung, beide zugleich verwirklichen zu wollen. Anders formuliert: Wenn Freiheit der Inhalt wie das Ziel der Philosophie ist, oder kantisch gesprochen, wenn die praktische Vernunft die Grundlage der Vernunft ist, muss dies Folgen für jede philosophische Darstellung haben. Aber welche?

Die erste Konsequenz einer praktischen Wende der Philosophie ist der Anspruch, verständlich zu sein. Diesen Anspruch erhebt ein Philosoph, der nicht nur die Wahrheit sucht und manchmal vielleicht sogar besitzt, sondern der vor allem die Freiheit im Visier hat. Die Allgemeinheit der Freiheit bekommt mit der Aufklärung den Vorrang vor der Allgemeinheit der Wahrheit bzw. der

12 Das übernimmt sogar Kant selbst: „Allein, wie viel und mit welcher Richtigkeit würden wir wohl denken, wenn wir nicht gleichsam in Gemeinschaft mit andern, denen wir unsere und die uns ihre Gedanken mitteilen, dächten!“ (Kant 1999: 58)

13 Heidegger 2015: 488. 
Wissenschaft. In anderen Worten: Es wird nicht mehr hauptsächlich die Wahrheit, sondern die Freiheit als leitende Idee der philosophischen Tätigkeit betrachtet. Nicht jeder muss natürlich die Kritik der Vernunft oder gar die Mathematik kennen, aber da das unter ihnen waltende Gesetz doch das Sittengesetz ist, sollte sie auch niemand ignorieren. Es ist der praktische Vorrang der Freiheit, der auf diese Weise die gelehrten Formen der Philosophie infrage stellt.

Wenn die Vernunft allgemein ist, muss sie sich konkret behaupten, allgemeingültig werden, sie gilt für jeden und jeder hat an ihr teil. Was für die Denker des Rationalismus noch abstrakt geblieben war, wurde allmählich zu einer Herausforderung für die Vernunft selbst. Diese allgemeine Bewegung lässt sich nirgends präzise verorten, sie behauptet sich aber zwischen Bayle oder Thomasius und Kant. Es wird indes kein Philosoph diesen Anspruch der Freiheit so perfekt zusammenfassen wie Rousseau, der nicht zufällig gerade Kant tief erschüttern sollte. Rousseau war kein Schulphilosoph, hat aber möglicherweise größere Wirkung gehabt als alle Schulphilosophen zusammen. Seine Botschaft war im Grunde die richtige. Daraus entstand Kants Problem: Wie kann man diese allgemeine Freiheit philosophisch begründen und allgemein mitteilen?

\section{Kant und die Forderungen der Freiheit}

Wie auf anderen Gebieten und in anderen Problemzusammenhängen ist auch hier die Rolle Kants zentral. Es ist üblich geworden, ihm eine Missachtung der Sprache vorzuwerfen, und zwar unter gleich drei Gesichtspunkten: als Vermittlung des Denkens: Kant habe nur die ,reine' Vernunft behandelt; des Stils: sein Ausdruck sei schlecht und größtenteils unverständlich; der Form der Philosophie: er habe sein eigenes Versprechen eines philosophischen Systems selbst nicht eingelöst. Es handelt sich in allen drei Fällen um voreilige Urteile, so sehr sie sich durch Wiederholung und Tradition auch gefestigt haben mögen.

Man könnte sogar ganz im Gegenteil zeigen, dass Kant die Zusammenführung der Welt- und der Schulphilosophie ohne Vermischungen und Verluste durchgeführt hat; dass er der Erfinder der deutschen Philosophie, d. h. der Philosophie in deutscher Sprache war und als solcher in die europäische Geschichtsschreibung auch eingegangen ist; ferner, dass er die Frage nach der Form der Philosophie als die Frage vom System und vom Zugang zum System überhaupt als Erster formuliert und als Problem betrachtet hat, ohne dafür vielleicht die passende Lösung gefunden zu haben. 
a) Kant ist es gelungen, die Diglossie von gelehrter und ,gemeiner' Sprache zu überwinden. Wie kann das sein, wo Kants Unfähigkeit zu einer verständlichen Rede doch geradezu sprichwörtlich geworden ist und ihm oft eine völlige Vernachlässigung der Rolle der Sprache für das Denken vorgeworfen wurde?

Einerseits gilt Kant als Purist der reinen Vernunft, was zu dem Einwand führte, er habe in seinen Kritiken die Sprache nicht mitbedacht. Früh kam dieses Urteil von Zeitgenossen wie Hamann, Herder, Hegel oder Humboldt auf, die Kant mit einer Metakritik konfrontierten, die als konkrete Basis der Kritik fungieren sollte, so wie die Sprache überhaupt erst die Basis des Denkens bilde. Dieser Protest gegen die Vernachlässigung einer genuin sprachlichen Vermittlung der Vernunft führte zu einer Auffassung der Philosophie als Sprachkritik, die in Auseinandersetzung mit Kant selbst traditionsbildend wurde.

Angesichts der Tatsache, dass Kant im Ruf stand (nicht zuletzt durch einen von Garve geäußerten Vorwurf), überhaupt nicht schreiben zu können, mag das paradox anmuten. Gleichwohl: Das Erstaunliche an Kant besteht darin, dass sich seit und mit ihm eine Versöhnung der gelehrten und der vermittelnden Sprache beobachten lässt. Er hat als Erster die Schulphilosophie der Weltphilosophie zugeordnet, ohne dabei jene dieser zu opfern, was ihn von der Popularphilosophie fundamental unterscheidet. Die Grundlegung der Metaphysik der Sitten ist eine meisterhafte Widerlegung dieser Vorwürfe: Diese klare und allgemein verständliche Schrift führt mustergültig in das System ein.

b) Andererseits wurde der erbärmliche Stil Kants beklagt, als wäre er ein geradezu sinnliches Zeichen für seine Vernachlässigung oder Geringschätzung der Sprache. Schon die Frühromantiker haben sich darüber lustig gemacht, und es ist für das Bild von Kant charakteristisch geblieben. Es ist sogar zum Topos geworden, dass Kant schlecht schreibt, dass er vielleicht sogar der erste Philosoph gewesen ist, der schlecht schreibt. Friedrich Schlegel, Jean Paul, auch Heine wollten es so sehen. ${ }^{14}$

Bevor man dies zu widerlegen versucht, scheint es angebracht, Beispiele für Kants schlechten Ruf genauer zu belegen. Solche finden sich zahlreich in dem Werk des englischen Dichters Thomas De Quincey, dem Kant so wichtig war, dass er sogar seine Autobiographie mit einer Auseinandersetzung mit ihm beschloss und Kants ,letzten Tagen', last days, eine eigene Schrift widmete. De Quincey schreibt: „Kant was a great man, but he was obtuse and deaf as an antediluvian boulder with regard to language and its capacities. “15 De Quinceys Erklärung dafür ist zunächst quantitativer Art; Kant schreibe zu lange Sätze:

14 Bis hin zu Nancy 1975.

15 De Quincey 1890b: 259. 
„He has sentences which have been measured by a carpenter, and some of them run two feet eight by six inches. “16 Dabei müsse der Leser den Faden verlieren, was seine Texte dunkel mache:

Parts so remote as the beginning and the end of such a sentence can have no sensible relation to each other: not much as regards their logic, but none at all as regards their more sensuous qualities - rythms, for instance, or the continuity of metaphor. ${ }^{17}$

An anderer Stelle heißt es:

A sentence is viewed by him, and by the most of his countrymen, as a rude mould or elastic form admitting of expansion to any possible extent: it is laid down as a rough outline, and then by superstruction and epi-struction it is gradually reared to a giddy altitude which no eye can follow. ${ }^{18}$

Bei solch langen Sätzen könne das Bewusstsein nicht mehr alles aufnehmen. Der Geist des Rezipienten zeigt sich schlechterdings überfordert. De Quincey spricht noch nicht von multitasking, wohl aber von overtasking. ${ }^{19}$ Dies verstößt gegen das psychologische Prinzip einer narrowness of mind, wie es von Locke etabliert worden war. ${ }^{20}$

Ist unter solchen Bedingungen eine Synthesis überhaupt noch möglich? De Quincey berührt hier den Kern der Kant'schen Lehre. Die Sätze, vor allem wenn sie so lang sind, bauen Synthesen auf. Aber sind sie wirklich zu lang?

Kant hat die Erfahrung als ein Lesen bezeichnet, das die Daten der Sinnlichkeit als Buchstaben zu einer Einheit erhebt. ${ }^{21}$ Die Sätze können zwar recht lang sein, wie De Quincey bemerkt, aber sie betrachten den jeweiligen Gegenstand unter einem besonderen Licht, schlagen ihn einer bestimmten Kategorie zu. Die Perspektivierung der Begriffe wird stets mitberücksichtigt. ${ }^{22}$ Die Frage ist immer: Was für eine Synthesis und was für eine Einheit lässt sich anstreben?

c) Diese Perspektivierung, die man oft bis in kleinste Verästelungen der Sätze nachweisen kann, ja diese Modalisierung der Sprache ist nicht der einzige Ertrag von Kants umfassender Leistung. Kant hat auch die verschiedenen philosophischen Diskurse perspektivisch betrachtet. Das Wechselspiel zwischen

16 Ebd.

17 Ebd.

18 De Quincey 1890a: 160.

19 „The mind finds itself overtasked“ (ebd.: 161).

20 Vgl. Locke 1975: II, 10.

21 Vgl. Kant 1998: A 314.

22 Diesen Aspekt hat das große Kant-Buch von Josef Simon sehr subtil akzentuiert, vgl. Simon 2003. 
dem Dogmatismus und dem Skeptizismus etwa hat er in seiner inneren Logik verfolgt. Es ist dies sogar eine mögliche Definition der Kritik: dass sie nach den Bedingungen der Möglichkeit philosophischer Diskurse fragt. Sie will selbst keine weitere Philosophie sein, sondern eine philosophische Reflexion der Bedingungen philosophischen Wissens.

Mehr noch: Kant fragt nach der Möglichkeit eines philosophischen Systems, das auf der Vernunft gründet und seine eigene Grundlage auch selbst zu erklären vermag. Das System kann nicht mehr nur objektiv und von außen beschrieben werden, wie etwa noch bei Spinoza, sondern muss in seinem Entstehen nachvollziehbar sein. Der Philosoph muss deswegen seine eigene Handlung auf- und nachweisen. Dieser Anspruch machte sich bereits in den Anfängen der neuzeitlichen Philosophie bei Descartes geltend, weil hier zum ersten Mal die Selbstvergewisserung des Geistes als Voraussetzung der ,Wahrheit' fungierte. Seitdem muss alles im Denken im Sinn einer zeitlichen Erfahrung produziert werden. Prinzipiell erhält eine solche Bestrebung jedoch erst ausgehend von der kantischen Philosophie ihren wirklichen Anstoß, und das aus mindestens einem wesentlichen Grund: Diese erlegt sich die Pflicht auf, die Subjektivität bei der Entstehung des Systems selbst zu berücksichtigen. Sie sieht dieses System nicht mehr als unmittelbar im Verstand gegeben an. Der Geist entdeckt die Formen der Dinge nicht mehr einfach in sich selbst und nicht einmal außerhalb von sich. Er muss sie durch eine Erfahrung hervorbringen. Er muss sich im Selbstbewusstsein erkennen und untrennbar damit verbunden auch die Welt erkennen, denn das eine ist nicht ohne das andere vorhanden.

Dies wurde spätestens mit dem Streit um Spinoza klar, den Jacobi gegen die Vernachlässigung der Subjektivität in der rationalistischen Philosophie der Ethica entfacht hatte. ${ }^{23}$ Die objektive Darstellung des Wissens im System der Kenntnisse würde stets einen blinden Fleck aufweisen, nämlich die Stelle der Subjektivität selbst, des geistigen Urhebers des Systems. Das angeblich systematische Wissen reflektierte sich selbst nicht angemessen, und dieses Defizit an Reflexivität ließ sich nicht mehr übersehen, sobald man Kant zugestand, dass kein ,Gegebenes“ als solches einen Ausgangspunkt für die Philosophie bilden könne, unabhängig von den (subjektiven) Bedingungen, unter denen dieses ,Gegebene‘ uns ,gegeben“ ist. Daher kann man die Ansicht vertreten, das Problem der Philosophie nach Kant sei durchaus die Frage nach einem System, das auf seine eigenen Möglichkeitsbedingungen zurückzugehen vermag, anders gesagt, das die subjektive Instanz einbeziehen und

23 Vgl. Jacobi 1998. 
legitimieren kann, indem es sie als Wissenssystem oder System des Absoluten darstellt. ${ }^{24}$ Eine derartige Versöhnung von System und Subjektivität besagt auch, dass der Leser (wieder) leichter den Zugang zum System findet: Das System sorgt nunmehr für seine eigene Pädagogik, die eine bessere Popularisierung verspricht.

Die kategorische Einbeziehung der subjektiven Tätigkeit im Aufbau der Erkenntnis hat Kant in seiner Lehre vom Schematismus dargestellt. In seiner eher technischen Bestimmung hat er die Funktion der Begriffe Form und Materie anerkannt, denn er sah in ihnen „zwei Begriffe, welche aller andern Reflexion zugrunde gelegt werden, so sehr sind sie mit jedem Gebrauch des Verstandes unzertrennlich verbunden“, wobei das eine die Bestimmung des anderen als bestimmbar bedeutet. ${ }^{25}$ Die Form geht aller Materie voran, die Erkenntnis ist an und für sich eine Formgebung des Mannigfaltigen. Dies ist die Wirkung des transzendentalen Schematismus, der die Formung des sinnlich Mannigfaltigen vollzieht, wobei er es in Übereinstimmung mit einer der Kategorien festlegt.

Die Form führt die Tätigkeit der Synthesis des Subjekts aus, indem sie die Begriffe verzeitlicht. Sie ermöglicht, dass sie im Kontext einer Erfahrung angewandt werden. Die Form in dieser technischen Bedeutung ist das ,Schema', also nicht das Bild, das empirisch und räumlich ist, sondern die Formungs- und Vorstellungsbewegung, das strukturierende Prinzip eines Bildes. Als Verzeitlichung führt das Schema eine differenzierte Einheit ein, die Regel, die ein Mannigfaltiges in die Zeit einordnet. Die Einbildungskraft produziert das Schema und ermöglicht das Eindringen des reinen Begriffs in das Sinnliche. Indem die Schematisierung seinen Umfang einschränkt, verschafft sie der Kategorie „Sinn und Bedeutung“, ${ }^{26}$ wobei die Form das bestimmbare Sinnliche bestimmt. Der Begriff wird versinnlicht, das Sinnliche wird nunmehr sinnvoll. Kant vergleicht das Schema mit einem „Monogramm der reinen Einbildungskraft a priori“, 27 das heißt mit einer Signatur oder einem Namenszug, die das Subjekt auf das Sinnliche setzt. Der reine Verstand (anders gesagt, die Subjektivität) drückt der Erfahrung seinen Stempel auf, indem er die Kategorien in dem doppelten Sinn, wie er sie für seinen Gebrauch benötigt und wie er sie in Bewegung setzt, mobilisiert. Das Allgemeine bestimmt so das Besondere. Doch bei dieser Formgebung, bei

24 Kant wurde freilich bald von Fichte wie von Hegel vorgeworfen, dass er die Rückführung der Inhalte des Philosophierens auf den Akt des Denkens nur ungenügend geleistet hätte, indem er die reinen Verstandesbegriffe als ,gegeben` anerkannte, statt sie herzuleiten.

25 Kant 1998: A 266/B 322. Zur die Vorgeschichte des Begriffspaars bei Kants Vorläufern vgl. Sgarbi 2010: 103-128; Tommasi 2008: $77 \mathrm{f}$.

26 Kant 1998: B 299.

27 Kant 1998: A 142/B 181; vgl. A 570/B 598, A 834/B 862. 
welcher der Verstand in die Sinnlichkeit eindringt, unterscheidet sich die letzte Einheit. Diese ermöglicht die Synthesis der Synthesen, die Vereinigung der Kategorien und des sinnlich Mannigfaltigen in einer einzigen Erfahrung. Kant bezeichnet sie als transzendentale Einheit der Apperzeption. Sie unterscheidet sich zwangsläufig von der durch die Synthesen produzierten Einheit. Der Schematismus der Kategorie der Quantität produziert die numerische Einheit des Objekts der Erfahrung, das als ,eines‘ erfasst wird. Aber die Kategorie lässt sich nur unter der Bedingung anwenden, dass sie in einem transzendentalen Akt, der diese Formgebung im Voraus ordnet, synthetisiert wird. Kant schlägt vor, die Einheit des Begriffs bei der Erkenntnis eines Objekts die qualitative Einheit zu nennen, nämlich, wie er erklärt, „die Einheit der Zusammenfassung des Mannigfaltigen, wie etwa die Einheit des Themas in einem Schauspiel, einer Rede, einer Fabel“ ${ }^{28}$

Das ist genau besehen eine erstaunliche Erklärung. Hat man sich das so vorzustellen, dass die Gliederung der Schematisierungen, die den Kategorien einen Sinn geben, sich nach den verborgenen Regeln einer transzendentalen thematischen inventio vollzieht? Der Philosoph würde dann die Anschauung der Zeit teilen, wie es der Rhetor oder der Dichter zu tun pflegen, wenn sie ihre Argumente erfinden und ihre Figuren - oder ihre Schemata - anordnen. Diese Dimension wird jedenfalls bei den späteren Philosophen wie bei den Romantikern ihren Widerhall finden. Die Bestimmung des Sinnlichen durch eine Form nach dem kantischen Modell des Schematismus beherrscht nicht nur die Frage nach der Bedeutung, denn die Begriffe erhalten erst einen Sinn, wenn sie sich versinnlichen, sondern auch die der Darstellung des Denkens in einer Form. Dabei stellt sich die Subjektivität dar, indem sie sich verzeitlicht, und gleichzeitig muss sie als sie selbst erkennbar sein. Kant hat damit eine neue Aufgabe für die philosophische Darstellung erfunden, die nicht er, sondern die Nachfolger $\mathrm{zu}$ erfüllen versuchten.

\section{Ausblicke}

Die Perspektivierung der philosophischen Diskurse sowie der Sprache nach Kant macht es sowohl möglich als auch zwingend, an verschiedenen Darstellungsformen zu experimentieren. Die Frühromantik tat dies radikal und erfreulich bunt, aber auch die zeitgenössischen Philosophen haben ihrerseits versucht, sich individuell möglichst verständlich $\mathrm{zu}$ machen. Auch sie haben Fragmente verfasst, wie Schleiermacher oder Hegel, oder Aphorismen, wie Schelling, die immer

28 Kant 1998: B 114. 
in einem ganz konkreten Verhältnis zu dem aufzubauenden System stehen; manche haben Dialoge geschrieben (Schelling, Solger), die teilweise auf einer Auseinandersetzung mit Platon beruhen, sich teilweise aber auch mit entsprechenden Versuchen der Aufklärung, wie denen Diderots, vergleichen lassen. Ihre komplexe Logik ist dabei oft auf die Beziehung zwischen Dialektik und Dialog zurückzuführen. Wichtig wurde auch das Experimentieren mit der Form des Epos: ein interessanter Fall wäre bei Schelling die Werkstatt der Weltalter, die aber nie zur Vollendung gebracht wurde. ${ }^{29}$ Hegel hat dagegen in seiner Phänomenologie des Geistes eine völlig neue Form erfunden, die man als solche würdigen sollte: Er hat diese Schrift ebenso als Einleitung in das System wie als Darstellung des Systems selbst konzipiert. ${ }^{30}$ Er hat also für zwei Sorten von Lesern gleichzeitig zu schreiben versucht, für die Anfänger, die noch der Form der ,Entgegensetzung‘, der ,Spaltung“, des Bewusstseins verhaftet sind, und für die, die bereits wissen, dass jede Gestalt vom Standpunkt des ,Geistes', der reflektierten Substanz, zu beurteilen ist. Selten hat man bemerkt, dass Hegels Ausführungen zum Epos in der Analyse des ,geistigen Kunstwerks‘ die Art und Weise darlegten, wie sein eigenes Buch angemessen zu lesen sei. Danach wird er sich nie wieder an eine solche Bearbeitung der Form wagen. Es blieb ein einmaliger Versuch, populär und philosophisch zugleich zu bleiben. Dieser Versuch wurde aber meist als gescheitert betrachtet, Hegel galt bald als der dunkle Philosoph par excellence: Skoteinos.

Muss und kann die Philosophie demnach überhaupt populär werden?

Die Fragestellung führt leicht zu Missverständnissen. Von ihr aus, in ihrer Differenziertheit, kann nichts beobachtet werden, was den Bedarf einer großen Öffentlichkeit für die philosophische Analyse begründen könnte. Philosophen versuchen mit Genauigkeit und einer möglichst scharfen Begrifflichkeit zu hoher Komplexität vorzustoßen. Sie wollen erst einmal verstehen und brauchen Zeit. Wer wird ihnen dabei wohl folgen (wollen)?

In der Neuzeit erlebt sich aber prinzipiell jeder Mensch als freies Wesen. Der Weltbegriff der Philosophie, der alles betrifft, was für jedermann notwendig interessant ist, fordert zu Recht, dass die Philosophie, in ihren wesentlichen Zügen, zugänglich bleibt. Die Universalität des freien Menschen erstreckt sich auf die Kunst, wenn alle Menschen Künstler sind (Schleiermacher), und auf die Ideen gleichermaßen, denn alle denken auch. Die Philosophie hat dies in der Aufklärung durch verschiedene Strategien und literarische Gattungen zu beweisen versucht. Mit und nach Kant erfand man eine Philosophie, die in der Gestalt des

29 Vgl. Schelling 2002.

30 Hegel 1980. 
Systems sich selbst und alles begründen wollte und dabei spekulative Akrobatik nie scheute. Trotz ihres oft esoterischen Anscheins bleiben die Schriften, die unter diesen Bedingungen geschrieben wurden, nicht selten dem Versuch verpflichtet, eine Art ,höherer Popularität‘ zu erlangen, wie Schlegel dies nannte. Es wurde mit verschiedenen Formen, Gattungen und Kommunikationsstrategien experimentiert, was für sich schon darauf schließen lässt, dass die Philosophie das Problem der Zugänglichkeit oder gar der Einführung nicht kategorisch verabschieden wollte. Die freie Bearbeitung verschiedener Darstellungsformen um 1800 zeugt von dem immer erneuerten Versuch, dem Denken bzw. Wissen einen adäquaten Ausdruck zu verleihen, und zwar im Hinblick sowohl auf die Leser als auch auf die jeweiligen philosophischen Anforderungen selbst.

Insofern kann man hier zwar keine radikal demokratische Wende der Philosophie erkennen, aber doch den leisen, versteckten Versuch, eine Art Popularisierung mit anderen Mitteln zu betreiben. Vielleicht scheitert jeder Versuch einer Popularisierung, aber das besagt keineswegs, dass die Philosophie es nicht wagen sollte, dass sie es sogar tun muss - aber ohne Eile ...

\section{Literatur}

Beck, Philippe \& Denis Thouard (Hrsg.) (1995): Popularité de la philosophie. Fontenay, Saint-Cloud: E.N.S. Éditions.

Blumenberg, Hans (1987): Das Lachen der Thrakerin. Frankfurt a.M.: Suhrkamp.

De Quincey, Thomas (1890a): Style. In: The Collected Writings of Thomas De Quincey. Hrsg. von David Masson. Bd. 10: Literary Theory and Criticism. Edinburgh: Black, 134-245.

De Quincey, Thomas (1890b): Language. In: The Collected Writings of Thomas De Quincey. Hrsg. von David Masson. Bd. 10: Literary Theory and Criticism. Edinburgh: Black, 246-263.

Diderot, Denis (1961): Pensées sur l'interprétation de la nature. In: CEuvres philosophiques. Textes établis avec introductions, bibliographies et notes. Hrsg. von Paul Vernière. Paris: Garnier.

Ernesti, Johann August (1767): De philosophia populari. In: Ders., Opuscula oratoria. Orationes, prolusiones et elogia. 2. Ausgabe. Editio secunda multis partibus auctior \& emendatior. Lugduni Batavorum: Luchtmans, 189-197.

Fichte, Johann Gottlieb (1801): Sonnenklarer Bericht an das größere Publikum über das eigentliche Wesen der neuen Philosophie. Ein Versuch, die Leser zum Verstehen zu zwingen. Berlin: Realschulbuchhandlung.

Garve, Christian (1796): Von der Popularität des Vortrags. In: Vermischte Aufsätze, welche einzeln oder in Zeitschriften erschienen sind. Neuherausgegeben und verbessert von Christian Garve. Erster Theil. Breslau: Wilhelm Gottlieb Korn, 331-358.

Garve, Christian (1798): Eigene Betrachtungen über die allgemeinen Grundsätze der Sittenlehre. Ein Anhang zu der Uebersicht der verschiednen Moralsysteme von Christian Garve. Breslau: Wilhelm Gottlieb Korn. 
Hegel, Georg Wilhelm Friedrich (1980): Phänomenologie des Geistes. In: Gesammelte Werke. Bd. 9. Hrsg. von Wolfgang Bonsiepen \& Reinhard Heede. Hamburg: Meiner.

Heidegger, Martin (2015): Anmerkungen V. In: Gesamtausgabe. Bd. 97, Abt. 4: Hinweise und Aufzeichnungen, Anmerkungen I-V (Schwarze Hefte 1942-1948). Hrsg. von Peter Trawny. Frankfurt a.M.: Vittorio Klostermann, 431-518.

Jacobi, Friedrich Heinrich (1998): Werke. Bd. 1: Schriften zum Spinozastreit. Hrsg. von Klaus Hammacher \& Irmgard-Maria Piske. Hamburg: Meiner.

Kant, Immanuel (1998): Kritik der reinen Vernunft. Nach der 1. und 2. Orig.-Ausg. hrsg. von Jens Timmermann. Mit einer Bibliogr. von Heiner Klemme. Hamburg: Meiner.

Kant, Immanuel (1999): Was heißt: sich im Denken orientieren? In: Ders., Was ist Aufklärung? Ausgewählte kleine Schriften. Hrsg. von Horst Brandt. Hamburg: Meiner, 45-61.

Kant, Immanuel (2009): Vorrede. In: Ders., Metaphysik der Sitten. Teil 1: Metaphysische Anfangsgründe der Rechtslehre. Hrsg. von Bernd Ludwig. Hamburg: Meiner.

Locke, John (1975): An Essay concerning Human Understanding. Hrsg. von Peter Harold Nidditch. Oxford: Clarendon.

Nancy, Jean-Luc (1975): Logodaedalus (Kant écrivain). In: Poétique 21, 24-52.

Platon (1958): Theaitetos. In: Sämtliche Werke. Bd. 4: Phaidros, Parmenides, Theaitetos, Sophistes. In der Übers. von Friedrich Schleiermacher mit der Stephanus-Numerierung hrsg. von Walter F. Otto, Ernesto Grassi \& Gert Plamböck. Hamburg: Rowohlt, 147-251.

Quintilian, Marcus Fabius (1997): Institutio oratoria, Lib. VIII. Hrsg. von Cesare Marco Calcante. Mailand: Rizzoli, 1256-1411.

Schelling, Friedrich Wilhelm Joseph (2002): Schellingiana. Bd. 13, 1-2: Weltalter-Fragmente. Hrsg. von Klaus Grotsch. Stuttgart-Bad Cannstatt: Frommann-Holzboog.

Sgarbi, Marco (2010): La Kritik der reinen Vernunft nel contesto della tradizione logica aristotelica. Hildesheim u.a.: Olms.

Simon, Josef (2003): Kant. Die fremde Vernunft und die Sprache der Philosophie. Berlin, New York: De Gruyter.

Thouard, Denis (2016): Geteilte Ideen. Philosophische Versuche, den Leser zum Verstehen zu bringen. Aus dem Franz. von Ulrich Kunzmann. Berlin: Matthes \& Seitz.

Tommasi, Francesco Valerio (2008): Philosophia transcendentalis. La questione antepredicativa e l'analogia tra la Scolastica e Kant. Florenz: Olschki. 